\title{
Ordering infinite utility streams: efficiency, continuity, and no impatience*
}

\author{
José Carlos R. Alcantud ${ }^{\dagger} \quad$ Ram Sewak Dubey \\ January 27, 2016
}

\begin{abstract}
We study two related versions of the no-impatience postulate in the context of transitive and reflexive relations on infinite utility streams which are not necessarily complete. Both are excluded by the traditional (weak) anonymity axiom. We show explicit social welfare relations satisfying Strong Pareto and the weaker version of noimpatience that are compatible with continuity in all the traditional topologies in this field. However the stronger version of no-impatience is violated by all lower semicontinuous (in the sup or Campbell topologies) social welfare relations satisfying the Weak Pareto axiom.
\end{abstract}

Keywords: Continuous social welfare relations, Infinite utility streams, Multi-utility representation, No Impatience, Richter-Peleg representation.

Journal of Economic Literature Classification Numbers: D70, D90.

\footnotetext{
${ }^{*}$ We thank the Editor and an anonymous referee of this journal for their comments and suggestions. This research is supported by the Spanish Ministerio de Economía y Competitividad under project ECO201231933 (J. C. R. Alcantud).

${ }^{\dagger}$ Corresponding author. Facultad de Economía y Empresa, Universidad de Salamanca, E 37008, Salamanca, Spain. Email: jcr@usal.es

${ }^{\star}$ Department of Economics and Finance, School of Business, Montclair State University, Montclair, NJ 07043, USA. Email: dubeyr@mail.montclair.edu
} 


\section{Introduction}

This paper is concerned with the impatience implications of continuous intertemporal relations that verify monotonicity properties but do not necessarily verify the completeness axiom. The impatience axiom manifests itself in the traditional present discounted utility criteria. Authors like Koopmans (1960) or Diamond (1965) soon showed that under some common assumptions, attractive principles like Strong Pareto come at the cost of a preference for advancing the timing of future satisfactions. Further studies contributed to clarify some basic facts about the impatience implications of Pareto axioms in the presence of either continuities or representability, cf., Burness (1973), Svensson (1980), Shinotsuka (1998), Banerjee and Mitra (2007), or Banerjee and Dubey (2013) among others. In the seminal Koopmans (1960), "if we accept the Pareto axiom, it is the postulate of a utility function that creates the mathematical difficulty that equal treatment of all generations is not possible": Banerjee and Mitra (2007, p. 237). Svensson (1980, Section 1) claims that “in Koopmans (1960) and Diamond (1965) 'impatience' was a consequence of the monotonicity and the continuity of an ordering". According to Shinotsuka (1998): "continuity of a preference relation in an infinite horizon setting implies some form of impatience or myopia. Diamond (1965) was one of the earliest to formalize this idea". Many forms of impatience are ruled out when anonymity axiom is present. These axioms embody intergenerational equity by imposing the equal treatment of the generations. Here we consider patience implications of the common Diamond's (weak) anonymity axiom. ${ }^{1}$

Suppose that we are interested in representable intertemporal preferences that verify the strongest form of the Pareto axiom. Then, not only they must contradict anonymity in extremely reduced program spaces (cf., Basu and Mitra (2003)), but also they must show impatience at uncountably many infinite streams (Banerjee and Mitra (2007, Theorem $1)$ ), and similar conclusions are valid when monotonicity is relaxed to Weak Pareto in broader domains (Banerjee and Dubey (2013, Theorem 1)). See also Alcantud (2012, 2013); Alcantud and García-Sanz (2013).

Suppose now the case of continuous intertemporal relations that verify the strongest form of the Pareto axiom, and let us consider the impatience consequences of referring continuity to the five topologies in Lauwers (1997)' analysis. Concerning possible violations of anonymity, the following facts provide a quite complete analysis. Diamond (1965) shows that continuity with respect to the Sup topology contradicts anonymity, thus the same is true for the product and the myopic topologies, which are coarser. Campbell (1985, Theorem 5) assures that lower semicontinuity with respect to the Campbell (therefore the product) topology contradicts anonymity. However, Svensson (1980) proves that

\footnotetext{
${ }^{1}$ Authors like Lauwers (1998) contribute to the analysis of extended versions of that principle that exclude more generic forms of impatience.
} 
there exist complete preorders - or social welfare orders- that are Strongly Paretian, anonymous, and continuous with respect to Svensson's topology. In this paper we investigate the impatience implications that result from these assumptions when the completeness axiom is dropped.

We define two versions of the usual no-impatience conditions that are successive weakenings of the anonymity axiom. They coincide for social welfare orders, although in general the two conditions are different. In Section 3 we prove that they have different implications too. We show that the weakest version (NIw) is compatible with the existence of Richter-Peleg representations that satisfy Strong Pareto, and that this is not the case of the other version (NIs), for very general forms of the program space. We observe a sharp contrast with Banerjee and Dubey (2010), who prove that there do not exist Strongly Paretian and anonymous intertemporal relations that have Richter-Peleg representations, respectively multi-utility representations with their set of utilities being countably infinite. More importantly, to prove that result we explicitly ${ }^{2}$ produce Strongly Paretian intertemporal relations, continuous in the product topology, that verify NIw and that have multi-utility representations continuous in the product topology, their set of utilities being countably infinite. In particular this means than when we drop the completeness axiom, a certain form of no-impatience is fully compatible with Strongly Paretian relations that are continuous in any of the aforementioned topologies, since the product topology is coarser than the Campbell, myopic, sup, or Svensson topologies. We also emphasize the contrast of our explicit construction with Banerjee and Dubey (2014, Theorem 2), which states that the existence of a social welfare order satisfying a relaxed form of Strong Pareto and noimpatience involves non-constructive techniques. Again, when we drop the completeness axiom a certain form of no-impatience leads to a different conclusion even if the full force of the Pareto axiom is invoked.

In Section 4 we concentrate on the more restrictive no-impatience (NIs) property, for which the conclusions are surprisingly different than in the case of the weaker version (NIw). Except for the case of the Svensson topology, we have a widespread incompatibility: when the Weak Pareto axiom is imposed on an intertemporal relation, lower semicontinuity in either the sup or the Campbell topology yields violations of no-impatience in the sense of NIs. This imposes the impossibility of representing such relations by multiutility representations that are lower semi-continuous in these topologies. However the

\footnotetext{
${ }^{2}$ Recent literature contains several contributions on the subject of explicit description of social welfare orders satisfying useful properties, following a conjecture by Fleurbaey and Michel (2003) that there exists no explicit description (that is, avoiding the axiom of choice or similar contrivances) of an ordering which satisfies the Anonymity and Weak Pareto axioms. Lauwers (2010) and Zame (2007) have shown (by using different definitions of "non-constructive" devices) that it is not possible to explicitly describe any social welfare order satisfying the Anonymity and Strong Pareto axioms.
} 
use of the Svensson topology yields a positive answer.

\section{Preliminaries}

\subsection{Notation}

Let $\mathbb{R}$ and $\mathbb{N}$ be the sets of real numbers and natural numbers respectively. Let $Y$, a nonempty subset of $\mathbb{R}$, be the set of all possible utilities that any generation can achieve. Then $X \equiv Y^{\mathbb{N}}$ is the set of all possible utility streams. If $x \in X$, then $x=\left(x_{1}, x_{2}, \cdots\right)$, where, for all $n \in \mathbb{N}, x_{n} \in Y$ represents the amount of utility that the generation of period $n$ earns. The sequence $(0,0,0, \cdots) \in X$ is denoted by 0 , and the sequence $(1,1,1, \cdots)$ is denoted by $e$.

If $Y$ has only one element, then $X$ is a singleton, and the problem of ranking or evaluating infinite utility streams is trivial. Thus, without further mention, the set $Y$ will always be assumed to have at least two distinct elements. For all $y, z \in X$, we write $y \geq z$ if $y_{n} \geq z_{n}$, for all $n \in \mathbb{N}$, we write $y>z$ if $y \geq z$ and $y \neq z$, and we write $y \gg z$ if $y_{i}>z_{i}$ for all $i \in \mathbb{N}$.

For $x \in X$, and $N \in \mathbb{N}$, we denote $\left(x_{1}, \cdots, x_{N}\right)$ by $x(N)$ and $\left(x_{N+1}, x_{N+2}, \cdots\right)$ by $x[N]$. Thus, given any $x \in X$ and $N \in \mathbb{N}$, we can write $x=(x(N), x[N])$. If $x, y \in X$, and $N \in \mathbb{N}$, we write $z=(x(N), y)$ to denote the element $z \in X$, satisfying $z_{k}=x_{k}$ for all $k \in\{1, \cdots, N\}$ and $z_{N+k}=y_{k}$ for all $k \in \mathbb{N}$.

If $x \in X$ and $\sum_{n=1}^{\infty} x_{n}<\infty$, we define $S(x)=\sum_{n=1}^{\infty} x_{n}$, and the sum of the truncated sequence $x(N)$ for any $N \in \mathbb{N}$ is denoted by $S(x(N))$; that is, $S(x(N))=\sum_{n=1}^{N} x_{n}$. The unit simplex in $X$ is the set $\operatorname{Sim}=\{x \in X: S(x)=1\}$. For $x, y \in X$, we will denote $\left(\left|x_{1}-y_{1}\right|,\left|x_{2}-y_{2}\right|, \cdots\right) \in \mathbb{R}_{+}^{\mathbb{N}}$ by $|x-y|$.

\subsection{Social Welfare Relations}

A social welfare relation (SWR), interchangeably called a preference relation on $X$, is a binary relation $\geqslant$ on $X$ which is reflexive (i.e., $x \geqslant x$ for any $x \in X$ ) and transitive (i.e., for any $x, y, z \in X, x \geqslant y$ and $y \geqslant z$ imply that $x \geqslant z$ holds). Given a preference relation $\geqslant$ on $X$, we indicate its asymmetric and symmetric parts by $>$ and $\sim$. Recall that for any $x, y \in X, x>y$ means $x \geqslant y$ and not $y \geqslant x$, and $x \sim y$ is defined as $x \geqslant y$ and $y \geqslant x$.

For the SWR $\geqslant$ on $X$, for each $x \in X$, we define the lower contour set $L C(x)$ and upper contour set $U C(x)$ as

$$
L C(x)=\{y \in X: x \geqslant y\} ; \text { and } U C(x)=\{y \in X: x \leqslant y\},
$$


respectively. We say that a $\mathrm{SWR} \geqslant$ on a topological space $(X ; \tau)$ is lower (respectively upper) semicontinuous if $L C(x)$ (respectively $U C(x)$ ), is a closed subset of $X$ for every $x \in X$. Also it is continuous if it is both upper and lower semicontinuous.

A social welfare order (SWO), interchangeably called a preference order on $X$, is a binary relation $\geqslant$ on $X$ which is complete (i.e., if for any $x, y \in X$ either $x \geqslant y$ or $y \geqslant x$ holds) and transitive. The social welfare order is representable (by a utility function) if there is some $u: X \rightarrow \mathbb{R}$ such that for any $x, y \in X$, we have

$$
x \geqslant y \Leftrightarrow u(x) \geq u(y) .
$$

We refer to this representation as social welfare function (SWF).

While it is obviously not possible to associate an incomplete social welfare relation $\geqslant$ a SWF, we may nevertheless find a function $v: X \rightarrow \mathbb{R}$ such that

$$
x \geqslant y \Rightarrow v(x) \geq v(y) \text {, and } x>y \Rightarrow v(x)>v(y) .
$$

We say that $v$ is a Richter-Peleg representation (RPR) of the SWR $\geqslant$ on $X$. If the $\mathrm{SWR} \geqslant$ has an RPR $v$ then, and only then, $\geqslant$ can be extended to a SWO represented by the SWF $v{ }^{3}$ However this generalized formulation of utility representation has the shortcoming that it is unable to capture the indecisiveness region of the SWR: when $v(x)>v(y)$ all we can deduce is that either $x>y$ or $x$ and $y$ are unrelated by $\geqslant$. This is the price to pay for dispensing with completeness while using a notion of 'utility function'.

Nevertheless we may still allow for incompleteness of a SWR and represent it by a set of functions without any information loss. The SWR $\geqslant$ on $X$ admits a multi-utility representation if there exists a family $V$ of functions $v: X \rightarrow \mathbb{R}$ such that

$$
x \geqslant y \Leftrightarrow[v(x) \geq v(y) \text { for all } v \in V] .
$$

This notion is of universal nature, because Evren and Ok (2011, Proposition 1) prove that any preorder (i.e., reflexive and transitive binary relation) has a trivial multi-utility representation. Alcantud et al. (2015, Remark 2.7) prove that for any preorder, the existence of a multi-utility representation with the set of utilities being countably infinite implies the existence of a Richter-Peleg representation.

\subsection{The Efficiency conditions}

We refer to the following efficiency conditions on the social welfare relations.

Definition 1. Strong Pareto (SP) : If $x, y \in X$ and $x>y$, then $x>y$.

Definition 2. Weak Pareto (WP) : If $x, y \in X$ and $x \gg y$, then $x>y$.

\footnotetext{
${ }^{3}$ The binary relation $R$ is an extension of $\geqslant$ when $\geqslant$ is a subset of $R$ and $>$ is a subset of the asymmetric part of $R$.
} 


\subsection{The No Impatience conditions}

Given $x \in X$, and $M, N \in \mathbb{N}$, we denote by $x(M, N)$ the sequence $x^{\prime} \in X$ defined by,

$$
x_{M}^{\prime}=x_{N}, x_{N}^{\prime}=x_{M} \text { and } x_{n}^{\prime}=x_{n}, \forall n \neq N, M .
$$

Observe that the definition of a new sequence $x(M, N)$ in (1) from some $x \in X$ involves swapping one-period utilities corresponding to periods $M$ and $N$, ceteris paribus.

With respect to equity we refer to the Anonymity axiom ${ }^{4}$ as follows:

Definition 3. Anonymity (AN): A finite permutation of a utility stream produces a utility stream with the same social utility, i.e., for all $x \in X$ and $M, N \in \mathbb{N}, x \sim x(M, N)$.

When this axiom is in conflict with other properties, one may at least try to impose weaker equity axioms. We investigate the case of no impatience. In order to define the pertinent concepts, let us first say that a social welfare relation $\geqslant$ exhibits impatience at $x \in X$, if there exist $M, N \in \mathbb{N}$ with $M>N$ such that either

$$
\text { (i) } x_{M}>x_{N} \text { and } x(M, N)>x \text {; or (ii) } x_{M}<x_{N} \text { and } x>x(M, N) \text {. }
$$

The impatience condition captures the intuition that the preference relation $\geqslant$ exhibits a preference towards "immediate gratification".

We provide two alternative definitions of lack of impatience (or no impatience) as below. They relax AN in the following terms:

Definition 4. No Impatience (weak) axiom (NIw): A social welfare relation $\geqslant$ is said to exhibit weak no impatience on $X$ if for each $x \in X$ and any $M, N \in \mathbb{N}$ with $M>N$, whenever $x_{M}>x_{N}$ we have that $x(M, N)>x$ is false, and $x_{M}<x_{N}$ implies $x(M, N)<x$ is false.

This definition does not require $x(M, N)$ and $x$ to be comparable. However, if $x(M, N)$ and $x$ are comparable, then

$$
x(M, N) \leqslant x \text { in case } x_{M}>x_{N} \text { and } x(M, N) \geqslant x \text { if } x_{M}<x_{N} .
$$

Definition 5. No Impatience (strong) axiom (NIs): A social welfare relation $\geqslant$ is said to exhibit strong no impatience on $X$ if for each $x \in X$ and any $M, N \in \mathbb{N}$ with $M>N$, whenever $x_{M}>x_{N}$ we have $x(M, N) \leqslant x$, and $x_{M}<x_{N}$ implies $x(M, N) \geqslant x$.

The weak no impatience axiom described in Definition 4 is weaker than the strong no impatience axiom in Definition 5. Proposition 1 below ensures that they are different. They coincide for preference orders, although Banerjee and Dubey (2014, Section 4) proves that even when we have Strongly Paretian preference orders, they do not imply AN.

\footnotetext{
${ }^{4}$ The concept of Anonymity was introduced in the social welfare literature by Diamond (1965) who described it as "equal treatment" of all generations (present and future).
} 


\subsection{Metric functions}

The following metrics are used to examine continuity properties of the social welfare relations in induced topologies.

Definition 6. Product Metric: For all $x, y \in X$,

$$
d(x, y)=\sum_{n \in \mathbb{N}} \frac{\left|x_{n}-y_{n}\right|}{2^{n}} .
$$

Definition 7. Sup Metric: For all $x, y \in X$,

$$
d(x, y)=\sup _{n \in \mathbb{N}}\left|x_{n}-y_{n}\right|
$$

Definition 8. Campbell Metric: For all $x, y \in X$,

$$
d(x, y)=\sup _{n \in \mathbb{N}} \frac{\delta\left(x_{n}, y_{n}\right)}{n}, \quad \text { where } \quad \delta\left(x_{n}, y_{n}\right)= \begin{cases}1, & \text { if } x_{n} \neq y_{n} \\ 0, & \text { otherwise. }\end{cases}
$$

Definition 9. Svensson Metric: $\quad$ For all $x, y \in X$,

$$
d(x, y)=\min \left\{1, \sum_{n=1}^{\infty}\left|x_{n}-y_{n}\right|\right\} .
$$

The following properties are satisfied by Svensson, sup and product metrics. ${ }^{5}$

(M.1) If $x, y \in X$, then $d(x, y)=d(|x-y|, 0)$.

(M.2) If $x, y \in X$ be such that $x \geq y$ then $d(x, 0) \geq d(y, 0)$.

(M.3) If $x \in X$ and $M \in \mathbb{N}$, then $d((0(M), x), 0) \leq d(x, 0)$.

(M.4) If $\lambda_{n} \in[0,1]$ for all $n \in \mathbb{N}$, and $\lim _{n \rightarrow \infty} \lambda_{n} \rightarrow 0$, then $\lim _{n \rightarrow \infty} d\left(\lambda_{n}(e(M), 0[M]), 0\right)=0$ for every $M \in \mathbb{N}$.

We denote the set of metric functions satisfying (M.1)-(M.4) by $\Delta$. The Campbell metric meets (M.1)-(M.3) but violates (M.4) whenever it is meaningful, because $d(\lambda(e(M), 0[M]), 0)=$ 1 for every $M \in \mathbb{N}$ and $\lambda>0$. Using metric $d \in \Delta$, function $f: X \rightarrow \mathbb{R}_{+}$defined as

$$
f(x) \equiv d(x, 0) \text { for all } x \in X
$$

\footnotetext{
${ }^{5}$ Readers are referred to Banerjee and Mitra (2008) for a detailed description of these properties. Observe that (M.4) is meaningful only in certain cases, e.g., when $[0, \varepsilon) \subseteq Y$ for some $\varepsilon>0$.
} 
(see Banerjee and Mitra (2008) for details) satisfies the sub-additivity property:

$$
\text { For any } x, y \in X \text {, with } x+y \in X, f(x+y) \leq f(x)+f(y) \text { holds. }
$$

Finally, for any metric $d$ we define the distance between a point $\bar{x} \in X$ and a set $A \subset X$ as

$$
d(\bar{x}, A)=\inf _{x \in A} d(x, \bar{x}) .
$$

\section{Multi-utility and Richter-Peleg representations}

Banerjee and Dubey (2010) ask whether one can define ethical (i.e., satisfying SP and AN) SWRs that can be represented by not just a single utility function but possibly many utility functions. However such attempt to avoid the impossibility result in Basu and Mitra (2003) failed to yield a positive response. Furthermore, their negative result for the representation of the social welfare orders persists even if anonymity is replaced by no impatience conditions. Banerjee and Mitra (2007, Theorem 1) proves that every social welfare function satisfying SP displays impatience at uncountably many utility streams on every non-trivial $X$. Banerjee and Dubey (2013, Theorem 1) show that the conclusion remains valid when SP is replaced by WP for $X=Y^{\mathbb{N}}$, if $Y$ contains subsets of order type similar to the set of negative and positive integers. This implies that every social welfare function satisfying WP must violate NIw (or NIs) on these domains. With this in view, we explore the possibility of nontrivial multi-utility representation of the social welfare relations satisfying SP and NIw (or NIs). We obtain very different conclusions depending on the version of the no-impatience postulate that we adopt.

\subsection{No Impatience as NIw}

We proceed to show that the situation is quite different if AN is relaxed to NIw. Proposition 1 below proves that if one weakens AN to no impatience as NIw (Definition 4), then instead of the negative results in Banerjee and Dubey (2010, Proposition 1, Theorem 2), a possibility result arises. Therefore continuity with respect to relevant topologies could be checked for. In fact we show that continuity with respect to the product topology can be imposed too. This ensures continuity with respect to the relevant topologies overviewed by Lauwers (1997) in his analysis of the extension to the infinite population case of the topological approach to social choice theory.

Proposition 1. For each $X=Y^{\mathbb{N}}$ with $\varnothing \neq Y \subseteq \mathbb{R}$, there exist SWRs $\geqslant$ on $X$ that satisfy SP and NIw (but not NIs). Further the SWRs have both multi-utility representations continuous with respect to the product topology (with the set of utilities being countably infinite), 
and Richter-Peleg representations ${ }^{6}$. Consequently, such SWRs are continuous with respect to the product topology.

Proof. Let $\mathbf{V}=\left\{v_{k}: k \in \mathbb{N}\right\}$ be the countable family of functions with $v_{k}: X \rightarrow \mathbb{R}$ given by $v_{k}(x)=\sum_{i=1}^{k} i x_{i}$. Define for any $x, y \in X$ :

$$
x \geqslant y \text { if and only if } v_{k}(x) \geqslant v_{k}(y) \text { for all } k \in \mathbb{N} \text {. }
$$

This produces a SWR on $X$ that verifies SP and by construction, $\geqslant$ has a multi-utility representation $\mathbf{V}$ with the set of utilities being countably infinite. Because $v_{k}$ is a projection and these are continuous and open with respect to the product topology (cf., Willard (1970, Theorem 8.6)), $v_{k}$ is continuous with respect to the product topology too. Let us check that $\geqslant$ verifies NIw.

For each $x \in X$ and $M, N \in \mathbb{N}$ with $M>N$, suppose $x_{M}>x_{N}$ (resp. $x_{N}>x_{M}$ ). Irrespective of the case,

$$
v_{k}(x)-v_{k}(x(M, N))=(M-N)\left(x_{M}-x_{N}\right) \text { if } k>M .
$$

We claim that $x(M, N) \geqslant x$ (respectively, $x \geqslant x(M, N))$ is false. To prove it we only need to observe that $v_{k}(x(M, N)) \geqslant v_{k}(x)$ (respectively $\left.v_{k}(x(M, N)) \leqslant v_{k}(x)\right)$, is false when $k>$ $M$. In order to prove that $\geqslant$ has Richter-Peleg representations we use the aforementioned Alcantud et al. (2015, Remark 2.7). Finally, to prove that $\geqslant$ does not verify NIs, we consider $x=\left(0,1,0_{\text {con }}\right)$ and $M=2, N=1$, then $x_{M}>x_{N}$ but $x(M, N)=\left(1,0_{\text {con }}\right) \leqslant x$ is false because $v_{1}(x(M, N))=1 \leqslant 0=v_{1}(x)$ is false.

Since $X$ is first countable in the product topology, it is not difficult to prove continuity of $\geqslant$ from the fact that each $v_{k}$ is continuous with respect to the product topology.

\subsection{No impatience as NIs}

In this subsection we show that the alternative definition NIs does not lead to positive results as described in Proposition 1 above. For this we first establish that the social welfare relation $\geqslant_{P}$ satisfying SP and NIs does not admit a Richter-Peleg representation for every non-trivial domains $X$.

Proposition 2. Assume that $Y$ contains two distinct elements. There does not exist a social welfare relation $\geqslant$ satisfying $S P$ and NIs that has a Richter-Peleg representation.

\footnotetext{
${ }^{6}$ Using Alcantud et al. (2015, Proposition 2.5), we can infer that the SWR $\geqslant$ also admits countable continuous strong multi-utility representation. A social welfare relation $\geqslant$ on $X$ has a strong multi-utility representation if there exists a multi-utility representation $V$ such that $x>y \Leftrightarrow[v(x)>v(y)$ for all $v \in V]$.
} 
Proof. The proof is by contradiction. We assume that $Y$ contains 0 and 1 for ease of exposition, instead of $a, b \in Y$ with $a<b$. Let $\geqslant_{P}$ be a SWR satisfying SP and NIs with its asymmetric and symmetric parts $>_{P}$ and $\sim_{P}$ respectively.

Let $v: X \rightarrow \mathbb{R}$ be a RPR of this SWR. We use this $v$ to construct the following SWO: For all $x, y \in X$, we define $\geq$ as

$$
x \geq y \text { if and only if } v(x) \geq v(y) .
$$

We show that the representable $\mathrm{SWO} \geq$ satisfies SP and NIs.

(a) SP: For any $x, y \in X$ satisfying $x>y$, using SP we get $x>_{P} y$. By the RPR of the SWR, we must then have $v(x)>v(y)$. Using the definition of $\geq$, it implies that $x>y$.

(b) NIs: Without loss of generality, let us assume that for any $x \in X$ and $M, N \in \mathbb{N}$ with $M>N$, we have $x_{M}>x_{N}$. Then by NIs, we have $x(M, N) \leqslant_{P} x$ and using the RPR $v(x(M, N)) \leq v(x)$. In other words, $v(x(M, N))>v(x)$ and $x(M, N)>_{P} x$, must be false. Thus $v(x(M, N)) \leq v(x)$ or $x(M, N) \leq x$.

Thus, we have established $\geq$ to be a SWO satisfying SP and NIs. Also the RPR $v$ is a representation of $\geq$.

While describing NIs, the utility sequence $x \in X$ was chosen arbitrarily. Hence, we have proved existence of the representation $v: X \rightarrow \mathbb{R}$ satisfying SP which does not violate NIs for any $x \in X$.

This contradicts a result in Banerjee and Mitra (2007, Theorem 1) where it has been proved that every representation $v: X \rightarrow \mathbb{R}$ of the SWO satisfying SP must violate the NIs at uncountably many $x \in X$ where none of these $x \in X$ are constant utility streams. The contradiction completes the proof of the Proposition.

We use the aforementioned Alcantud et al. (2015, Remark 2.7) to prove that $\geqslant_{P}$ satisfying SP and NIs cannot have a multi-utility representation with the set of utilities being countably infinite and thereby establish our claim in the beginning of this sub-section.

Remark 1. Propositions 1 and 2 together immediately suggest: Does there exist a social welfare relation satisfying SP and NIs that has a multi-utility representation continuous with respect to the product topology? This would help to clarify the possible distinction between existence of non-trivial multi-utility representations and Richter-Peleg representations in the analysis of infinite utility streams. We address this issue later on in Section 4. 


\section{Continuity properties of the Social Welfare Relations}

We can draw another consequence from Proposition 1. Banerjee and Dubey (2014, Theorem 2), shows that the existence of a SWO satisfying SP and NIs involves use of nonconstructive techniques. This is also true if SP is relaxed to the infinite Pareto ${ }^{7}$. Proposition 1 shows that their conclusion crucially hinges on completeness in the sense that if we dispense with completeness, then one can explicitly define SWRs that satisfy SP and NIw without the appeal to the Axiom of Choice. As shown in the Proposition 1, these SWRs have the additional useful property of being continuous in product topology.

In this section we examine the continuity properties of the SWRs satisfying SP and NIs, as well as implications regarding their representability by multi-utilities. Observe that NIs is more restrictive compared to NIw, as it requires $x(M, N)$ to be comparable to $x$. This difference could be important because the introduction of the additional comparability requirements on $\gtrsim$ (making it more complete than the SWR $\geqslant$ ) sometimes causes incompatibilities in the intergenerational analysis.

On the positive side, we note that an example in Banerjee and Dubey (2014, Section 4) provides an explicit description of SWR $\gtrsim$ satisfying SP and NIs. ${ }^{8}$ Even though it uses the family of functions $v_{k}: X \rightarrow \mathbb{R}$ similar to those used in Proposition 1, the following example shows that the two SWRs are distinct.

Example 1. Let us check that $\geqslant$ in Proposition 1 is not a subset of $\gtrsim$. Consider

$$
x=\left(\frac{1}{1^{2}}, 1-\frac{1}{1 \cdot 2}, \frac{1}{3^{2}}, 1-\frac{1}{3 \cdot 4}, \frac{1}{5^{2}}, 1-\frac{1}{5 \cdot 6}, \cdots\right)
$$

and $y=(0,1,0,1,0,1,0, \cdots)$. Then $x \geqslant y$ because for each even $k, v_{k}(x)-v_{k}(y)=0$ and for each odd $k, v_{k}(x)-v_{k}(y)=\frac{1}{k}$. However neither $x>y$ nor $x \sim y$ hold true.

It is important to note that the SWR $\gtrsim$ determines the ranking of any pair of utilities by only looking at the tail of the family of functions $v_{k}(\cdot)$, or in other words, it must ignore the initial $v_{k}(\cdot)$ of finite length.

Henceforth we assume that $X=[0,1]^{\mathbb{N}}$. This domain permits to make continuities and related properties meaningful.

\footnotetext{
${ }^{7}$ Infinite Pareto is defined as follows: For $x, y \in X$, if $x>y$, and $x_{i}>y_{i}$ for infinitely many $i \in \mathbb{N}$, then $x>y$.

${ }^{8}$ Using the countable family of functions, $v_{k}: X \rightarrow \mathbb{R}$, given by $v_{k}(x)=\sum_{i=1}^{k} i x_{i}$, it defines

$$
x>y \text { if and only if } \exists N \text { such that } v_{k}(x)>v_{k}(y) \forall k \geq N,
$$
}

and

$$
x \sim y \text { if and only if } \exists N \text { such that } v_{k}(x)=v_{k}(y) \forall k \geq N,
$$

and let $x \gtrsim y$ if and only if $x>y$ or $x \sim y$. 


\subsection{WP and NIs axioms}

In this subsection we explore the possibility of combining WP and NIs for lower semicontinuous social welfare relations.

\subsubsection{Sup Topology}

In the next result we show that the SWR satisfying WP and NIs does not satisfy continuity in the sup topology.

Proposition 3. There does not exist a social welfare relation satisfying WP, NIs and lower semi - continuity in the sup topology.

Proof. The proof is by contradiction. We assume that there exists a SWR $\geqslant$ satisfying WP, NIs, and lower semi -continuity in the sup topology. Let

$u(n) \equiv\left(\frac{4^{n}-1}{4^{n}}, \frac{4^{n}-2}{4^{n}}, \cdots, \frac{2}{4^{n}}, \frac{1}{4^{n}}\right)$ and $\bar{u}(n) \equiv\left(\frac{4^{n}-1}{4^{n}}+\frac{1}{4^{n+1}}, \frac{4^{n}-2}{4^{n}}+\frac{1}{4^{n+1}}, \cdots, \frac{1}{4^{n}}+\frac{1}{4^{n+1}}\right)$

for all $n \in \mathbb{N}$. For $p \in \mathbb{N}$, let $r(p)$ denote the first non-zero remainder of the successive divisions of $p$ by 4 , and $q(p)$ the number of divisions with a zero remainder [For example, $r(52)=1$ and $q(52)=1$.]. We define

$$
u^{\prime}(n+1) \equiv\left(\frac{4^{n+1}-1}{4^{n+1}}, \cdots, \frac{4 p+3}{4^{n+1}}, \cdots, \frac{4 p+2}{4^{n+1}}, \frac{4 p}{4^{n+1}}, \frac{4 p}{4^{n+1}}, \cdots, \frac{1}{4^{n+1}}\right) \text { for } n \in \mathbb{N},
$$

where $p$ runs from 1 to $4^{n}-1$, and the term $\frac{4 p}{4^{n+1}}$ is repeated $q(4 p)$ times if $r(4 p)=1$, and $q(4 p)+1$ times otherwise. Note that

(a) every element of $\bar{u}(n)$ is contained in $u(n+1)$ for all $n \in \mathbb{N}$,

(b) $u^{\prime}(2)$ is obtained by postponing elements of $u(1)$ and preponing higher elements available in $u(2)$ to the corresponding positions in $\bar{u}(1)$,

(c) for all $n>1, u^{\prime}(n+1)$ is obtained in two steps:

Step 1 Postpone elements of $u^{\prime}(n)$ and prepone higher elements available in $u(n+1)$ to the corresponding positions in $\bar{u}(n)$. This gives $u^{\prime \prime}(n+1)$ which contains repeated elements, which are not placed at consecutive locations. 
Step 2 We postpone each of the repeated elements, occurring at the first instance, and prepone higher elements so that the repeated elements are placed at consecutive locations to obtain $u^{\prime}(n+1)^{9}$.

Consider the utility streams ${ }^{10}$

$$
\begin{gathered}
x^{0}=(u(1), u(2), \cdots), \quad y=(\bar{u}(1), \bar{u}(2), \cdots), \text { and } \\
x^{n}=\left(\bar{u}(1), \cdots, \bar{u}(n), u^{\prime}(n+1), u(n+2), \cdots\right) \text { for all } n \in \mathbb{N} .
\end{gathered}
$$

Observe that the utility stream $x^{n}$ is obtained from $x^{n-1}$ by preponing finitely many higher utilities from future dates to earlier dates. Since the social welfare order satisfies NIs, we get $x^{1} \leqslant x^{0}$ and $x^{n} \leqslant x^{n-1}$ for all $n \in \mathbb{N}$. Also, ${ }^{11}$

$$
\left[\sup _{t \in \mathbb{N}}\left|x_{t}^{n}-y_{t}\right|=\frac{n}{4^{n+1}}+\frac{1}{4^{n+2}} \text { for all } n \in \mathbb{N}\right] \rightarrow \lim _{n \rightarrow \infty} x^{n}=y .
$$

Since SWR $\geqslant$ satisfies lower semi-continuity in sup topology, we get $y \leqslant x^{0}$. However, $y_{t}>x_{t}^{0}$ for all $t \in \mathbb{N}$ which leads to $y>x^{0}$ by WP. This contradiction completes the proof.

\subsubsection{Campbell Topology}

Subsequent to the positive results on continuity in Svensson (1980), the continuity of ethical social welfare relations attracted wide attention. In one early contribution, Campbell (1985) proposed a topology (since named as Campbell topology) and showed that with reference to it, the impossibility results of Diamond (1965) persist. In the following proposition we examine the continuity of SWRs satisfying NIs and WP in Campbell's topology.

Proposition 4. There does not exist a social welfare relation satisfying WP, NIs and lower semi-continuity in the Campbell topology.

Proof. The proof is by contradiction. We assume that there exists a SWR $\geqslant$ satisfying WP, NIs, and continuity in the Campbell topology. Let

$$
x=\left(\frac{1}{2}, 1-\frac{1}{2^{2}}, 1-\frac{1}{2^{3}}, \cdots\right), \quad y=x[1]=\left(1-\frac{1}{2^{2}}, 1-\frac{1}{2^{3}}, \cdots\right) \gg x .
$$

\footnotetext{
${ }^{9}$ For illustration, in $u^{\prime}(2)=\left(\frac{15}{4^{2}}, \frac{14}{4^{2}}, \frac{12}{4^{2}}, \frac{12}{4^{2}}, \frac{11}{4^{2}}, \frac{10}{4^{2}}, \frac{8}{4^{2}}, \frac{8}{4^{2}}, \frac{7}{4^{2}}, \frac{6}{4^{2}}, \frac{4}{4^{2}}, \frac{4}{4^{2}}, \frac{3}{4^{2}}, \frac{2}{4^{2}}, \frac{1}{4^{2}}\right)$, each of the $4^{\text {th }}, 8^{\text {th }}$ and $12^{\text {th }}$ elements is the same as its immediate predecessor. The third and fourth elements of $u^{\prime}(2)$ are postponed to become $12^{\text {th }}$ and $16^{\text {th }}$ elements of $u^{\prime \prime}(3)$ in Step 1. In Step 2, the $12^{\text {th }}$ element of $u^{\prime \prime}(3)$ is postponed to become the $15^{\text {th }}$ element of $u^{\prime}(3)$.

${ }^{10}$ Similar utility streams have been used in Fleurbaey and Michel (2003, p. 796) and Basu and Mitra (2007, Theorem 4).

${ }^{11}$ For example, for $x^{1}, \sup _{t \in \mathbb{N}}\left|x_{t}^{1}-y_{t}\right|=\frac{1}{4^{2}}+\frac{1}{4^{3}}$ which is attained at $t=6,10,14$.
} 
Consider the utility streams

$$
x^{n}=\left(y(n), \frac{1}{2}, x[n+1]\right) \text { for all } n \in \mathbb{N} .
$$

Observe that the utility stream $x^{n}$ is obtained from $x^{n-1}$ by swapping one higher utility (the $(n+1)$-th term with value $\left.1-\frac{1}{2^{n+1}}\right)$ with an earlier lower utility (the $n$-th term with value $\frac{1}{2}$ ). Since the social welfare order satisfies NIs, we get $x^{1} \leqslant x$ and $x^{n} \leqslant x^{n-1}$ for all $n=2,3, \cdots$. Also, the fact that

$$
y-x^{n}=(\overbrace{0, \cdots, 0}^{n \text { terms }}, 1-\frac{1}{2^{n+2}}-\frac{1}{2}, \cdots) \quad \text { for all } n \in \mathbb{N}
$$

ensures

$$
\sup _{t \in \mathbb{N}} \frac{\delta\left(x_{t}^{n}, y_{t}\right)}{t}=\frac{1}{n+1} \text { for all } n \in \mathbb{N}
$$

which means that $\left\{x^{n}\right\}_{n \in \mathbb{N}}$ converges to $y$ in the Campbell topology. Since $\geqslant$ satisfies lower semi-continuity we get $x \geqslant y$. However, $y \gg x$ leads to $y>x$ by WP. This contradiction completes the proof.

\subsection{Topology for continuous Social Welfare Relations}

Having encountered impossibility of social welfare relations satisfying NIs and WP (which also implies similar outcome in case we use stronger efficiency notion of SP) in sup topology or Campbell topology, we explore the topology under which continuity can be restored. For the SWRs satisfying AN and SP, Banerjee and Mitra (2008) have obtained necessary and sufficient conditions which a topology should satisfy to ensure lower semicontinuity. The required condition is that the distance from the origin to the unit simplex in the space of utility streams be positive. In this subsection we intend to examine if their arguments are applicable for SWRs satisfying NIs and SP.

Proposition 5 establishes a necessary condition for the lower semicontinuity of the SWR satisfying NIs and SP. The proof closely follows the argument in Banerjee and Mitra (2008, Proposition 1).

Proposition 5. There does not exist a social welfare relation satisfying SP, NIs and lower semicontinuity for metric $d \in \Delta$ if $d(0, \operatorname{Sim})=0$.

Proof. By contradiction. We assume that there exists a SWR $\geqslant$ satisfying SP, NIs and lower semicontinuity in the topology induced by $d \in \Delta$ although $d(0, \operatorname{Sim})=0$. Then there 
exists a sequence $\left\{z^{N}\right\}_{N=1}^{\infty}, z^{N} \in \operatorname{Sim}$ for all $N \in \mathbb{N}$ and $d\left(z^{N}, 0\right) \rightarrow 0$ as $N \rightarrow \infty$. For each $N \in \mathbb{N}$, using the fact that $S\left(z^{N}\right)=1$, we can choose $k(N)$, the minimum integer such that the following inequality holds:

$$
S\left(z^{N}(k(N))\right)=\sum_{n=1}^{k(N)} z_{n}^{N} \geq 1-\frac{1}{N} .
$$

Denote $a(N)=S\left(z^{N}(k(N))\right)$ and define the vector $y(N)=\left(y_{1}(N), y_{2}(N), \cdots, y_{k(N)}(N)\right)$ as follows:

$$
\begin{aligned}
y_{1}(N) & =z_{1}^{N}+z_{2}^{N}+\cdots+z_{k(N)}^{N}=a(N), \\
y_{2}(N) & =z_{2}^{N}+z_{3}^{N}+\cdots+z_{k(N)}^{N}, \\
\cdots & \\
y_{k(N)-1}(N) & =z_{k(N)-1}^{N}+z_{k(N)}^{N}, \\
y_{k(N)}(N) & =z_{k(N)}^{N} .
\end{aligned}
$$

Observe that $\left|y_{m+1}(N)-y_{m}(N)\right|=z_{m}^{N}$ for all $m=1,2, \cdots, k(N)-1$ holds. Define $x \in X$ and $\bar{x} \in X$ as below:

$$
\begin{aligned}
& x=(0, y(1), 0, y(2), 0, y(3), \cdots), \text { and } \\
& \bar{x}=(1, y(1), 0, y(2), 0, y(3), \cdots) .
\end{aligned}
$$

We then define a utility stream $x^{N}$ for each $N \in \mathbb{N}$ using the utility stream $x$ in two steps.

Step 1 We interchange the element $y_{1}(N)$ in the first position of $y(N)$ and the first element $x_{1}=0$. After this interchange, $y(N)$ is transformed into a new sequence (renamed as $\left.y^{\prime}(N)\right)$

$$
y^{\prime}(N)=\left(0, y_{2}(N), \cdots, y_{k(N)}(N)\right) \text {. }
$$

Step 2 Next, beginning with second element of $y^{\prime}(N)$, each element of $y^{\prime}(N)$ is preponed by one period to obtain $y^{\prime \prime}(N)=\left(y_{2}(N), \cdots, y_{k(N)}(N), 0\right)$ and

$$
x^{N}=\left(a(N), y(1), 0, y(2), 0, \cdots, 0, y(N-1), 0, y_{2}(N), \cdots, y_{k(N)}(N), 0,0, y(N+1), 0, y(N+2), 0, \cdots\right) .
$$

Observe that in each of the two steps, we have preponed higher utility to an earlier period and there are finitely many substitutions for each $x^{N}$. Since $\geqslant$ satisfies NIs, $x \geqslant x^{N}$ must 
hold true for each $N \in \mathbb{N}$. Also for each $N \in \mathbb{N}$,

$$
\begin{aligned}
d\left(x^{N}, \bar{x}\right) & =f\left(1-a(N), 0,0, \cdots, z_{1}^{N}, z_{2}^{N}, \cdots, z_{k(N)-1}^{N}, z_{k(N)}^{N}, 0,0, \cdots\right) \\
& \leq f(1-a(N), 0, \cdots)+f\left(0,0, \cdots, z_{1}^{N}, z_{2}^{N}, \cdots, z_{k(N)-1}^{N}, z_{k(N)}^{N}, 0,0, \cdots\right) \\
& \leq f\left(\frac{1}{N}, 0,0, \cdots\right)+f\left(0,0, \cdots, z_{1}^{N}, z_{2}^{N}, \cdots, z_{k(N)-1}^{N}, z_{k(N)}^{N}, 0,0, \cdots\right) \\
& \leq f\left(\frac{1}{N}, 0,0, \cdots\right)+f\left(z_{1}^{N}, z_{2}^{N}, \cdots, z_{k(N)-1}^{N}, z_{k(N)}^{N}, 0,0, \cdots\right) \\
& \leq f\left(\frac{1}{N}, 0,0, \cdots\right)+f\left(z^{N}\right)
\end{aligned}
$$

the first line in (8) following from (M.1), the second line from (7), the third line from (M.2) and (8), the fourth line from (M.3), and the last line from (M.2). Thus using (M.4) we have:

$$
\lim _{N \rightarrow \infty} d\left(x^{N}, \bar{x}\right) \rightarrow 0 .
$$

Since $x^{N} \in L C(x)$ for all $N \in \mathbb{N}$, (9) implies that $\bar{x} \in L C(x)$, by lower semi-continuity. However, $\bar{x}_{1}=1>0=x_{1}$ and $\bar{x} \geq x$ lead to $\bar{x}>x$ by SP. This contradiction completes the proof of the Proposition.

Remark 2. Both the product and the sup metrics satisfy the condition $d(0, \operatorname{Sim})=0$ (cf., Banerjee and Mitra (2008, p. 6)). Hence, Proposition 5 implies that no social welfare relation, satisfying SP, NIs, and lower semi-continuity with respect to the sup topology, exists. This is already implied by Proposition 3.

Banerjee and Mitra (2008, Proposition 2) establish that for any metric $d \in \Delta$ satisfying the condition $d(0, \operatorname{Sim})>0$, there exists a social welfare order on $X=[0,1]^{\mathbb{N}}$ that verifies the SP, AN and continuity. Of course, such consequence continues to hold when the AN is weakened to NIs. Combining this fact and Proposition 5, the following theorem obtains.

Theorem 1. Let us select $d \in \Delta$, and denote by $\tau_{d}$ the topology induced by $d$. Then, there are social welfare relations satisfying SP, NIs, and lower semicontinuity in the $\tau_{d}$ topology if and only if $d(0$, Sim $)>0$. In this case, there are social welfare orders satisfying SP, NIs, and lower semicontinuity in the $\tau_{d}$ topology.

If we now invoke Banerjee and Mitra (2008, Lemma 2), which proves that the $\tau_{d}$ topology is larger than the Svensson topology when $d \in \Delta$ and $d(0, \operatorname{Sim})>0$, we can conclude as in their Theorem 2.

Corollary 1. Among all metrics $d \in \Delta$, the Svensson metric induces the smallest topology under which there exist lower semicontinuous social welfare relations satisfying SP and NIs. 


\subsection{Multi-utilities and continuity}

The results in this section have direct implications on the possibility of representing SWRs by multi-utilities with continuity properties, that we proceed to explore.

It is easy to check that with respect to any topology $\tau$, every SWR that has a $\tau$ continuous (resp., lower semicontinuous, upper semicontinuous) multi-utility representation is $\tau$-continuous (resp., lower semicontinuous, upper semicontinuous): cf., Evren and Ok (2011, p. 556). This natural property permits to solve a question posed in subsection 3.2 in the following terms:

Corollary 2. There exists no social welfare relation satisfying WP and NIs that has multiutility representations lower semicontinuous in the Campbell, sup, or product topologies.

Proof. The existence of any such representation implies the existence of SWRs satisfying WP, NIs, and lower semicontinuity in the Campbell, sup, or product topologies. The first instance contradicts Proposition 4. The other instances contradict Proposition 3.

In contrast to this negative fact, the recourse to the Svensson topology permits to give a quite positive answer to the same question:

Corollary 3. There exist social welfare orders satisfying SP and AN (thus NIs) that have multi-utility representations lower semicontinuous in the Svensson topology.

Proof. Svensson (1980) ensures the existence of a SWO satisfying SP and AN (thus NIs) that is continuous in the Svensson topology. An appeal to Evren and Ok (2011, Prop. 2) permits to conclude: it assures that for every lower semicontinuous preorder on a topological space, there exist lower semicontinuous multi-utility representations.

Remark 3. We cannot take full advantage of the continuity property of the SWO in the argument above, because it is not always true that continuous orders have continuous multi-utility representations: cf., Evren and Ok(2011, subsection 3.1). In fact we do not yet know if there are SP and NIs social welfare relations that have multi-utility representations continuous in the Svensson topology. Nevertheless, our results in subsection 3.2 prove that any such possible representation should consist of uncountably many utilities. 


\section{Conclusions}

In this paper we explore the implications of imposing completeness on efficient social welfare relations that do not display impatience.

If completeness is insisted upon, both SP as well as its weaker version WP are inconsistent with no impatience in the case of lower semi-continuous (with respect to sup or Campbell topology) social welfare relations. In addition, among a certain class of metric topologies, the Svensson topology is the smallest topology for which completeness is compatible with continuous social welfare relations not exhibiting any impatience and that are sensitive to the interests of any single generation.

However, if we dispense with completeness then we have a more involved situation. In order to discuss its implications under no impatience, we refer to two kinds of representability properties, namely, multi-utility representations -which always exist and thus are only useful if they verify additional requirements- and Richter-Peleg representations. Assume the SP axiom. Then a suitable form of the no impatience principle (NIw) is consistent with explicitly defined social welfare relations admitting multi-utility representations with the set of utilities being countably infinite (thereby admitting Richter-Peleg representations) which are continuous even in the product topology. Despite this, we conclude that in the context of evaluating infinite utility streams, the problems of finding potentially useful multi-utility representations and Richter-Peleg representation are significantly different. This comes from a comparison between Corollary 3 and Proposition 2: a stronger form of the no impatience principle (NIs) is inconsistent with Richter-Peleg representations but permits meaningful multi-utility representations (because we can impose that the utilities incorporate lower semi-continuity in the Svensson's topology). This confirms that for no impatient and incomplete relations, the property of having a Richter-Peleg representation seems to be more demanding than the non-trivial property of having lower semi-continuous multi-utility representations.

These results contribute to demonstrate that insistence on the completeness axiom should require careful consideration. 


\section{References}

Alcantud, J. C. R., 2012. Liberal approaches to ranking infinite utility streams: when can we avoid interference? Social Choice and Welfare 41 (2), 381-396.

Alcantud, J. C. R., 2013. The impossibility of social evaluations of infinite streams with strict inequality aversion. Economic Theory Bulletin 1 (2), 123-130.

Alcantud, J. C. R., Bosi, G., Zuanon, M., 2015. Richter-Peleg multi-utility representations of preorders. Theory and Decision, $1-8$.

Alcantud, J. C. R., García-Sanz, M. D., 2013. Evaluations of infinite utility streams: Pareto efficient and egalitarian axiomatics. Metroeconomica 64 (3), 432-447.

Banerjee, K., Dubey, R. S., 2010. On multi-utility representation of equitable intergenerational preferences. In: Basu, B., Chakravarty, S. R., Chakrabarti, B. K., Gangopadhyay, K. (Eds.), Econophysics and Economics of Games, Social Choices and Quantitative Techniques. Springer, pp. 175-180.

Banerjee, K., Dubey, R. S., 2013. Impatience implications of weakly Paretian orders: Existence and genericity. Journal of Mathematical Economics 49 (2), 134-140.

Banerjee, K., Dubey, R. S., 2014. Do all constructive strongly monotone intertemporal orders exhibit impatience? Journal of Mathematical Economics 52, 66-69.

Banerjee, K., Mitra, T., 2007. On the impatience implications of Paretian social welfare functions. Journal of Mathematical Economics 43 (3-4), 236-248.

Banerjee, K., Mitra, T., 2008. On the continuity of ethical social welfare orders on infinite utility streams. Social Choice and Welfare 30, 1-12.

Basu, K., Mitra, T., 2003. Aggregating infinite utility streams with intergenerational equity: The impossibility of being Paretian. Econometrica 71 (5), 1557-1563.

Basu, K., Mitra, T., 2007. Possibility theorems for aggregating infinite utility streams equitably. In: Roemer, J., Suzumura, K. (Eds.), Intergenerational Equity and Sustainability. (Palgrave) Macmillan, pp. 69-74.

Burness, H. S., 1973. Impatience and the preferene for advancement in the timing of satisfactions. Journal of Economic Theory 6, 495-507.

Campbell, D. E., 1985. Impossibility theorems and infinite horizon planning. Social Choice and Welfare 2 (4), 283-293.

Diamond, P., 1965. The evaluation of infinite utility streams. Econometrica 33 (1), 170-177.

Evren, Ö., Ok, E. A., 2011. On the multi-utility representation of preference relations. Journal of Mathematical Economics 47, 554-563.

Fleurbaey, M., Michel, P., 2003. Intertemporal equity and the extension of the Ramsey criterion. 
Journal of Mathematical Economics 39 (7), 777-802.

Koopmans, T., 1960. Stationary ordinal utility and impatience. Econometrica 28 (2), 287-309.

Lauwers, L., 1997. Continuity and equity with infinite horizons. Social Choice and Welfare 14 (2), 345-356.

Lauwers, L., 1998. Intertemporal objective functions - strong Pareto versus anonymity. Mathematical Social Sciences 35 (1), 37-55.

Lauwers, L., 2010. Ordering infinite utility streams comes at the cost of a non-Ramsey set. Journal of Mathematical Economics 46 (1), 32-37.

Shinotsuka, T., 1998. Equity, continuity, and myopia: a generalization of Diamond's impossibility theorem. Social Choice and Welfare 15 (1), 21-30.

Svensson, L., 1980. Equity among generations. Econometrica 48 (5), 1251-1256.

Willard, S., 1970. General Topology. Addison-Wesley.

Zame, W., 2007. Can utilitarianism be operationalized? Theoretical Economics 2, 187-202. 